\title{
Association of T-wave abnormalities with major cardiovascular events in diabetes: the ACCORD trial
}

\author{
Steven J. Mould ${ }^{1}$ (D) E Elsayed Z. Soliman ${ }^{1,2} \cdot$ Alain G. Bertoni $^{3} \cdot$ Prashant D. Bhave $^{1} \cdot$ Joseph Yeboah $^{1}$. \\ Matthew J. Singleton ${ }^{1}$
}

Received: 8 July 2020 / Accepted: 15 October 2020 / Published online: 8 January 2021

(C) Springer-Verlag GmbH Germany, part of Springer Nature 2021

\begin{abstract}
Aims/hypothesis T-wave abnormalities (TWA) are often found on ECG and signify abnormal ventricular repolarisation. While TWA have been shown to be associated with subclinical atherosclerosis, the relationship between TWA and hard cardiovascular endpoints is less clear and may differ in the presence of diabetes, so we sought to explore these associations in participants from the Action to Control Cardiovascular Risk in Diabetes (ACCORD) trial.

Methods TWA were operationally defined as the presence of any Minnesota Codes 5-1 through 5-4 in any lead distribution. Multivariable Cox proportional hazards models were constructed to examine relationships between TWA and clinical cardiovascular events. Secondary analyses explored the risks conferred by major vs minor TWA, differential effects of TWA by anatomic localisation (anterolateral, inferior or anterior lead distributions), and differing associations in those with or without prevalent CVD. Results Among 8176 eligible participants (mean 62.1 \pm 6.3 SD years, 61.4\% male), there were 3759 cardiovascular events, including 1430 deaths (473 of a cardiovascular aetiology), 474 heart failure events, 1452 major CHD events and 403 strokes. Participants with TWA had increased risks of all-cause mortality (HR 1.45 [95\% CI 1.30, 1.62], $p<0.0001$ ), cardiovascular mortality (HR 1.93 [1.59, 2.34], $p=0.0001$ ), congestive heart failure (HR $2.04[1.69,2.48], p<0.0001)$ and major CHD (HR $1.40[1.26,1.57], p<0.0001)$, but no increased risk of stroke (HR 0.99 [0.80, 1.23], $p=0.95)$. Major TWA conferred a higher risk than minor TWA. When TWA were added to the UK Prospective Diabetes Study risk engine, there was improved discrimination for incident CHD events, but only for those with prevalent CVD (area under the receiver operating characteristic curve 0.5744 and 0.6030 with $p=0.0067$ ). Adding TWA to the risk engine yielded improvements in reclassification that were of greater magnitude in those with prevalent CVD (net reclassification improvement [NRI] 0.24 [95\% CI 0.16, 0.32] in those with prevalent CVD, NRI 0.14 [95\% CI 0.07, 0.22] in those without prevalent CVD).

Conclusions/interpretation The presence and magnitude of TWA are associated with increased risk of clinical cardiovascular events and mortality in individuals with diabetes and may have value in refining risk, particularly in those with prevalent CVD.
\end{abstract}

Keywords Biomarkers $\cdot$ Diabetes $\cdot$ T-wave abnormalities

Matthew J. Singleton

mjsingle@wakehealth.edu

1 Section of Cardiology, Department of Internal Medicine, Wake Forest School of Medicine, Winston-Salem, NC, USA

2 Epidemiological Cardiology Research Center, Wake Forest School of Medicine, Winston-Salem, NC, USA

3 Department of Epidemiology and Prevention, Wake Forest School of Medicine, Winston-Salem, NC, USA

$\begin{array}{ll}\text { Abbreviations } \\ \text { ACCORD } & \begin{array}{l}\text { Action to Control Cardiovascular Risk in } \\ \text { Diabetes } \\ \text { CHF }\end{array} \\ \text { Congestive heart failure } \\ \text { MC } & \text { Minnesota Code } \\ \text { NRI } & \text { Net reclassification improvement } \\ \text { RI } & \text { Reclassification index } \\ \text { ROC } & \text { Receiver operating characteristic } \\ \text { TWA } & \text { T-wave abnormalities } \\ \text { UKPDS } & \text { UK Prospective Diabetes Study }\end{array}$




\section{Research in context}

\section{What is already known about this subject?}

- T-wave abnormalities (TWA) are a common ECG abnormality encountered during routine clinical practice, with an estimated prevalence of $1.3 \%$ of the general population

- In the absence of known clinical cardiovascular disease, these TWA are often non-specific and of an uncertain significance to clinicians

- While TWA have been shown to be associated with subclinical atherosclerosis, the relationship between TWA and hard cardiovascular endpoints is less clear and may differ in the presence of diabetes

\section{What is the key question?}

- What is the relationship between TWA and cardiovascular events in individuals with diabetes?

\section{What are the new findings?}

- Patients with TWA had increased risk of all-cause mortality, cardiovascular mortality, congestive heart failure and major coronary artery disease

How might this impact on clinical practice in the foreseeable future?

- The presence and magnitude of TWA may reflect subclinical CVD and may have value as a prognostic indicator of increased risk of clinical cardiovascular events in those with diabetes

\section{Introduction}

T-wave abnormalities (TWA) are a common ECG abnormality encountered during routine clinical practice with an estimated prevalence of $1.3 \%$ of the general population $[1,2]$. In the USA, the prevalence is $1.7 \%$ among black people and $1.1 \%$ among white people [3, 4]. The majority of these patients lack a cardiac history or discernible cause for these abnormalities [5, 6]. In the absence of known clinical cardiovascular disease, these TWA are often non-specific and of an uncertain significance to clinicians. While major ECG abnormalities such as ST segment elevations and Q-waves have been shown to be associated with subsequent clinical cardiovascular events [7], the prognostic importance of TWA has not been fully established. This importance of TWA is particularly uncertain in individuals with no history of clinical CVD who are found to have incidental TWA on routine ECG. Much of the previously published research on TWA has been limited to select populations with prior cardiac pathologies. In these studies, TWA was found to be associated with increased risk of all-cause mortality and cardiac mortality in the setting of ST elevation myocardial infarction, arrhythmia or heart failure. Among patients with diabetes, there is limited data about the prognostic significance of TWA. Therefore, our aim was to explore the relationship between TWA and cardiovascular events in individuals with diabetes, as this may represent a cost-effective and non-invasive way of screening for cardiac disease. To this end, we used patient-level data from the Action to Control Cardiovascular Risk in Diabetes
(ACCORD) trial and the ACCORDION follow-up study to investigate this relationship between TWA and subsequent clinical cardiovascular events.

\section{Methods}

Study design and population The ACCORD trial (NCT00000620) is a multicentre randomised clinical trial of diabetes and CVD across the USA and Canada. Individuals recruited for ACCORD had type 2 diabetes mellitus with an $\mathrm{HbA}_{1 \mathrm{c}}$ level of at least $9.4 \mathrm{mmol} / \mathrm{mol}(7.5 \%)$ and either age $\geq 40$ years with CVD or age $\geq 55$ years with atherosclerosis, albuminuria, left ventricular hypertrophy, or two or more additional risk factors from the following set: dyslipidaemia, hypertension, smoking or obesity. Patients were excluded if BMI was greater than $45 \mathrm{~kg} / \mathrm{m}^{2}$, serum creatinine level was greater than $132.63 \mu \mathrm{mol} / \mathrm{l}$, or if other serious illnesses were present.

The trial enrolled 10,251 participants who were randomly assigned into groups to assess strategies of glycaemic control, BP control and lipid control using a factorial design. Patients were provided with diabetes education as well as diabetes medications and glucose testing supplies. Participants randomised to the intensive therapy group attended monthly visits for 4 months and then every other month thereafter. Patients in the standard therapy groups were seen every 4 months. The study was approved by institutional review boards at each centre as well as the National Heart, Lung, 
and Blood Institute. All participants provided written informed consent.

Additionally, 5518 ACCORD participants were also enrolled in the lipid arm of the trial and further randomised to receive either simvastatin plus fenofibrate or simvastatin plus placebo. The remaining 4733 ACCORD participants were randomised to the BP control arm of the study. These individuals were randomised to either intensive BP control (systolic BP $<120 \mathrm{mmHg}$ ) or standard BP control (systolic $\mathrm{BP}<140 \mathrm{mmHg}$ ). At the end of the original ACCORD trial, the remaining participants were invited to enrol in the longterm surveillance study ACCORDION. In this follow-up study, the original participants continued to be followed for up to 14 years from the start of ACCORD.

Of the 10,251 ACCORD participants, 46 participants were excluded for absent baseline ECG. An additional 974 participants were excluded for baseline ECG that was of inadequate quality for analysis of the T-wave. Among those with baseline ECG of adequate quality for T-wave analysis, a total of 1055 participants were excluded for a concomitant overriding ECG abnormality that precludes T-wave analysis, including complete heart block, Wolff-Parkinson-White pre-excitation pattern, the presence of an artificial pacemaker, non-specific intraventricular conduction delay, complete left bundle branch block or complete right bundle branch block, leaving 8176 eligible participants. Demographic characteristics of those ACCORD participants who were included vs excluded are provided in electronic supplementary material (ESM) Table 1.

Exposure variables At the start of the ACCORD study, each participant self-reported their medical history. According to the study protocol, participants had a baseline ECG at the start of the trial. ECG was performed by trained electrocardiographers following a standardised protocol on a GE Marquette (Milwaukee, WI, USA) MAC 1200 electrocardiograph at a sampling rate of $500 \mathrm{~Hz}$. Once obtained, ECGs were digitally transmitted to the Epidemiological Cardiology Research Center (EPICARE) at Wake Forest School of Medicine, Winston-Salem, NC, USA. The ECGs were then manually checked for quality and automatically processed using the GE Marquette 12-SL program version 2001. We operationally defined TWA as the presence of any Minnesota Codes (MC) 5-1 through 5-4 in any lead distribution (ESM Table 2). For our sensitivity analysis exploring major vs minor TWA, major TWA were defined as any MC 5.1 or 5.2 in any lead distribution, while minor TWA were defined as any MC 5.3 or 5.4 in any lead distribution in the absence of any major TWA. For our analysis of the relationship between the anatomic localisation of TWA and the risk of adverse events, three binary variables were added to the model that corresponded to the presence or absence of TWA in the anterolateral leads (I, aVL, V6), inferior leads (II, III, aVF) and anterior leads (V2-V5).
Table 1 Baseline characteristics of eligible ACCORD study participants $(n=8176)$

\begin{tabular}{|c|c|c|c|}
\hline \multirow[t]{2}{*}{ Characteristic } & \multicolumn{2}{|l|}{ TWA } & \multirow[t]{2}{*}{$p$ value } \\
\hline & $\begin{array}{l}\text { Absent } \\
n=5728(70.1 \%)\end{array}$ & $\begin{array}{l}\text { Present } \\
n=2448(29.9 \%)\end{array}$ & \\
\hline Age (years) & $62.1 \pm 6.3$ & $63.4 \pm 6.9$ & $<0.000$ \\
\hline Sex (\% male) & 61.4 & 57.4 & 0.0008 \\
\hline Race (\% white) & 63.1 & 59.3 & 0.001 \\
\hline Years with diabetes & $10.3 \pm 7.3$ & $11.4 \pm 7.9$ & $<0.000$ \\
\hline Smoking, current (\%) & 14.2 & 14.5 & 0.74 \\
\hline History of CVD (\%) & 27.4 & 47.7 & $<0.000$ \\
\hline Weight (kg) & $93.6 \pm 18.5$ & $92.8 \pm 18.2$ & 0.08 \\
\hline Height (cm) & $170.3 \pm 9.8$ & $169.1 \pm 9.9$ & $<0.000$ \\
\hline Waist circumference $(\mathrm{cm})$ & $106.5 \pm 13.7$ & $106.9 \pm 13.6$ & 0.21 \\
\hline BMI $\left(\mathrm{kg} / \mathrm{m}^{2}\right)$ & $32.2 \pm 5.4$ & $32.4 \pm 5.4$ & 0.08 \\
\hline Systolic BP (mmHg) & $135.0 \pm 16.3$ & $139.6 \pm 18.4$ & $<0.000$ \\
\hline Diastolic BP (mmHg) & $75.1 \pm 10.2$ & $74.9 \pm 11.3$ & 0.52 \\
\hline Heart rate & $73.0 \pm 11.5$ & $71.9 \pm 12.0$ & 0.000 \\
\hline Total cholesterol (mmol/l) & $4.76 \pm 1.06$ & $4.79 \pm 1.10$ & 0.22 \\
\hline $\mathrm{HDL}(\mathrm{mmol} / \mathrm{l})$ & $1.09 \pm 0.30$ & $1.07 \pm 0.28$ & 0.007 \\
\hline LDL (mmol/l) & $2.72 \pm 0.87$ & $2.75 \pm 0.89$ & 0.20 \\
\hline VLDL (mmol/l) & $0.94 \pm 0.62$ & $0.97 \pm 0.62$ & 0.10 \\
\hline Triacylglycerols (mmol/l) & $2.14 \pm 1.64$ & $2.19 \pm 1.60$ & 0.27 \\
\hline $\begin{array}{l}\mathrm{HbA}_{1 \mathrm{c}}(\mathrm{mmol} / \mathrm{mol}) \\
\mathrm{HbA}_{1 \mathrm{c}}(\%)\end{array}$ & $\begin{array}{l}67 \pm 11.4 \\
8.25 \pm 1.04\end{array}$ & $\begin{array}{l}68 \pm 11.9 \\
8.40 \pm 1.09\end{array}$ & $<0.000$ \\
\hline Creatinine $(\mu \mathrm{mol} / \mathrm{l})$ & $78.68 \pm 19.45$ & $81.33 \pm 21.22$ & $<0.000$ \\
\hline
\end{tabular}

Continuous variables described as mean $\pm \mathrm{SD}$, categorical variables described as frequency $(\%)$

${ }^{a} p$ value as calculated by ANOVA for continuous and $\chi^{2}$ for categorical variables

Outcome variables Outcomes of interest were all-cause mortality, cardiovascular mortality, congestive heart failure (CHF), major CHD (a composite of death from CHD, nonfatal myocardial infarction and unstable angina) and stroke.

Statistical methods Baseline characteristics of our study population with and without TWA were compared using mean \pm SD for continuous variables and frequency (percentage) for categorical variables. Kaplan-Meier plots were used to compare the proportion of participants in each group who had an event during follow-up. The logrank test was used to compare groups on the Kaplan-Meier analysis. After ensuring that there was no evidence of substantial departures from the assumption of proportionality of hazards using the Martingale residual plots, we utilised Cox proportional hazards models to examine the relationships between the presence of TWA and subsequent clinical cardiovascular events. Our initial analysis (Model 1) was adjusted for age, sex, race and treatment assignment. Model 2 was adjusted for the covariates in Model 1, with the addition of history of CVD, smoking, years 
with diabetes, $\mathrm{BMI}$, and time-averaged systolic $\mathrm{BP}$, total cholesterol, HDL-cholesterol and $\mathrm{HbA}_{1 \mathrm{c}}$. Model 3 was adjusted for the covariates in Model 2, with the addition of ST segment abnormalities (which were operationalised as a three-level ordinal variable with possible values being no ST abnormalities [MC 4.0], minor ST abnormalities [any MC 4.3 or 4.4 in the absence of any MC 4.1 or 4.2], or major ST abnormalities [any MC 4.1 or 4.2]) and major Q-wave (any MC 1.1 or 1.2). Our fully adjusted model was used for our analysis of the risk conferred by minor vs major TWA and our supplemental analyses of the differential effects of TWA by anatomic lead group and the interaction between prevalent CVD at baseline and TWA. The incremental value of TWA in prediction of major CHD events was assessed by comparing the area under the receiver operating characteristic (ROC) curves for the UK Prospective Diabetes Study (UKPDS) [8] risk engine with and without the addition of TWA, according to the method of DeLong [9]. Two-sided $p$ values below 0.05 were considered to be statistically significant. Improvement in classification was assessed using the net reclassification improvement (NRI) index. Of the 8176 participants in the analysis, 90 were excluded in the NRI analysis due to missing data required for the UKPDS risk engine, leaving 8086 participants. All statistical analyses were conducted at Wake Forest School of Medicine using SAS version 9.4 (Cary, NC, USA).

\section{Results}

The characteristics of the study population are provided in Table 1. Baseline TWA were absent in 5728 participants

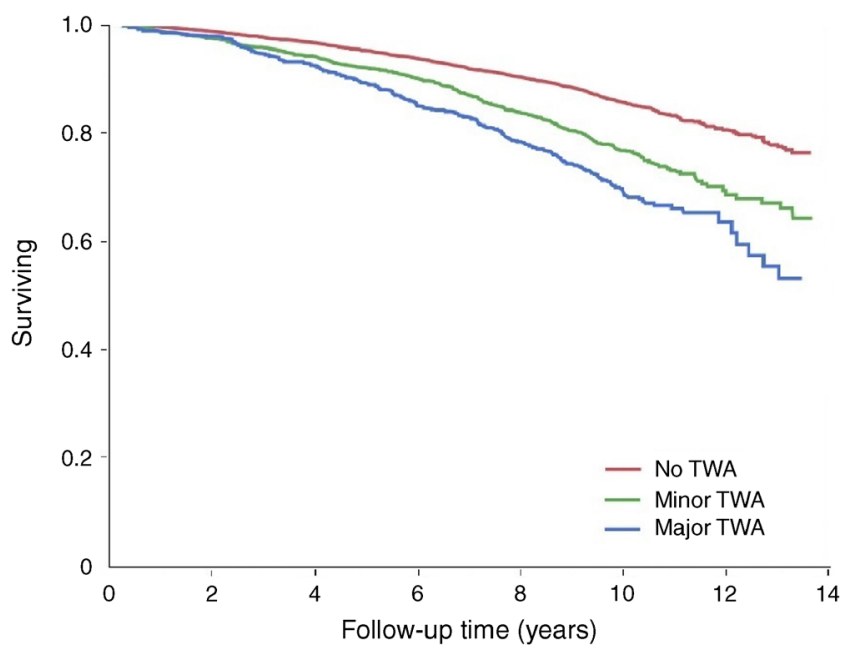

Fig. 1 All-cause mortality of ACCORD participants, stratified by TWA. Compared with those with no TWA, participants with minor TWA had a higher risk of death (logrank $p$ values for comparisons $p<0.001$ ). Similarly, those with major TWA had an even higher risk of death $(p<$ $0.001)$

and present in 2448 participants. Those with TWA were older, more likely to have a history of CVD, had a longer history of diabetes, higher mean systolic BP, and tended to have higher $\mathrm{HbA}_{1 \mathrm{c}}$.

In these 8176 eligible participants over a combined 75,145 person-years (median 9.4 [IQR 8.3-10.6]) of follow-up, there were 3759 cardiovascular events. Figure 1 illustrates the Kaplan-Meier survival curves of those with no TWA, minor TWA and major TWA. Table 2 depicts the association of baseline TWA with subsequent adverse events. Participants with TWA had increased risks of all-cause mortality (HR

Table 2 TWA at baseline and risk of adverse events

\begin{tabular}{|c|c|c|c|c|c|c|c|}
\hline \multirow[t]{2}{*}{ Outcome variable } & \multicolumn{2}{|c|}{$\begin{array}{l}\text { Participants with } \\
\text { endpoints }\end{array}$} & \multicolumn{2}{|c|}{$\begin{array}{l}\text { Absolute risk - events per } 1000 \text { person- } \\
\text { years }\end{array}$} & \multirow{2}{*}{$\begin{array}{l}\text { Model } 1 \\
\text { HR }(95 \% \mathrm{CI}) \\
p \text { value }\end{array}$} & \multirow{2}{*}{$\begin{array}{l}\text { Model } 2 \\
\mathrm{HR}(95 \% \mathrm{CI}) \\
p \text { value }\end{array}$} & \multirow{2}{*}{$\begin{array}{l}\text { Model } 3 \\
\text { HR }(95 \% \mathrm{CI}) \\
p \text { value }\end{array}$} \\
\hline & $\begin{array}{l}\text { No TWA } \\
n=5728\end{array}$ & $\begin{array}{l}\text { TWA } \\
n=2448\end{array}$ & No TWA & TWA & & & \\
\hline All-cause mortality & 814 & 616 & 14.7 & 28.3 & $\begin{array}{l}1.78(1.60,1.98) \\
p<0.0001\end{array}$ & $\begin{array}{l}1.46(1.31,1.64) \\
p<0.0001\end{array}$ & $\begin{array}{l}1.45(1.30,1.62) \\
p<0.0001\end{array}$ \\
\hline Cardiovascular mortality & 230 & 243 & 4.2 & 11.2 & $\begin{array}{l}2.58(2.15,3.09) \\
p<0.0001\end{array}$ & $\begin{array}{l}1.93(1.60,2.34) \\
p<0.0001\end{array}$ & $\begin{array}{l}1.93(1.59,2.34) \\
p<0.0001\end{array}$ \\
\hline $\mathrm{CHF}$ & 217 & 257 & 3.9 & 11.8 & $\begin{array}{l}2.99(2.49,3.58) \\
p<0.0001\end{array}$ & $\begin{array}{l}2.10(1.73,2.53) \\
p<0.0001\end{array}$ & $\begin{array}{l}2.04(1.69,2.48) \\
p<0.0001\end{array}$ \\
\hline Major CHD & 847 & 605 & 15.3 & 27.8 & $\begin{array}{l}1.85(1.67,2.06) \\
p<0.0001\end{array}$ & $\begin{array}{l}1.42(1.27,1.58) \\
p<0.0001\end{array}$ & $\begin{array}{l}1.40(1.26,1.57) \\
p<0.0001\end{array}$ \\
\hline Stroke & 260 & 143 & 4.7 & 6.6 & $\begin{array}{l}1.32(1.08,1.62) \\
p=0.008\end{array}$ & $\begin{array}{l}1.01(0.81,1.25) \\
p=0.94\end{array}$ & $\begin{array}{l}0.99(0.80,1.23) \\
p=0.95\end{array}$ \\
\hline
\end{tabular}

Model 1 adjusts for age, sex, race and treatment assignment

Model 2 adjusts for the covariates in Model 1, plus history of CVD, smoking, years with diabetes, BMI, creatinine, and time-averaged systolic BP, total cholesterol, $\mathrm{HDL}$ and $\mathrm{HbA}_{1 \mathrm{c}}$

Model 3 adjusts for the covariates in Model 2, plus ST segment abnormalities and major Q-wave 
Table 3 Major vs minor TWA at baseline and risk of adverse events

\begin{tabular}{|c|c|c|c|c|c|c|}
\hline \multirow[t]{2}{*}{ Outcome variable } & \multicolumn{2}{|c|}{$\begin{array}{l}\text { No TWA } \\
(n=5728)\end{array}$} & \multicolumn{2}{|c|}{$\begin{array}{l}\text { Minor TWA } \\
(n=1674)\end{array}$} & \multicolumn{2}{|c|}{$\begin{array}{l}\text { Major TWA } \\
(n=774)\end{array}$} \\
\hline & Events & $\begin{array}{l}\mathrm{HR}(95 \% \mathrm{CI}) \\
p \text { value }\end{array}$ & Events & $\begin{array}{l}\mathrm{HR}(95 \% \mathrm{CI}) \\
p \text { value }\end{array}$ & Events & $\begin{array}{l}\mathrm{HR}(95 \% \mathrm{CI}) \\
p \text { value }\end{array}$ \\
\hline All-cause mortality & 814 & Reference & 387 & $\begin{array}{l}1.37(1.20,1.55) \\
p<0.0001\end{array}$ & 229 & $\begin{array}{l}1.65(1.41,1.92) \\
p<0.0001\end{array}$ \\
\hline Cardiovascular mortality & 230 & Reference & 132 & $\begin{array}{l}1.62(1.30,2.02) \\
p<0.0001\end{array}$ & 111 & $\begin{array}{l}2.59(2.03,3.30) \\
p<0.0001\end{array}$ \\
\hline $\mathrm{CHF}$ & 217 & Reference & 143 & $\begin{array}{l}1.72(1.38,2.15) \\
p<0.0001\end{array}$ & 114 & $\begin{array}{l}2.76(2.16,3.52) \\
p<0.0001\end{array}$ \\
\hline Major CHD & 847 & Reference & 373 & $\begin{array}{l}1.34(1.18,1.52) \\
p<0.0001\end{array}$ & 232 & $\begin{array}{l}1.53(1.31,1.79) \\
p<0.0001\end{array}$ \\
\hline Stroke & 260 & Reference & 98 & $\begin{array}{l}0.83(0.58,1.19) \\
p=0.68\end{array}$ & 45 & $\begin{array}{l}0.88(0.63,1.22) \\
p=0.44\end{array}$ \\
\hline
\end{tabular}

Model is fully adjusted, including age, sex, race, treatment assignment, history of CVD, smoking, years with diabetes, BMI, creatinine, time-averaged systolic $\mathrm{BP}$, total cholesterol, $\mathrm{HDL}$ and $\mathrm{HbA}_{1 \mathrm{c}}$, ST segment abnormalities and major Q-wave

Minor TWA - any MC 5.3 or 5.4 in the absence of MC 5.1 or 5.2, in any anatomic distribution

Major TWA - any MC 5.1 or 5.2 in any anatomic distribution

1.45 [95\% CI 1.30, 1.62], $p<0.0001)$, cardiovascular mortality (HR 1.93 [1.59, 2.34], $p<0.0001$ ), CHF (HR 2.04 [1.69, $2.48], p<0.0001)$ and major CHD (HR 1.40 [1.26, 1.57], $p<0.0001$ ), but no increased risk of stroke (HR 0.99 [0.80, 1.23 ], $p=0.95)$.

We then conducted an exploratory analysis to examine the magnitude of TWA and risk of adverse events. Compared with the reference group of participants with no TWA $(n=$ 5728), participants with minor TWA $(n=1674)$ had increased risk of all-cause mortality, cardiovascular mortality, $\mathrm{CHF}$ and major CHD. Similarly, participants with major TWA $(n=$ 774) had even higher risk of all-cause mortality, cardiovascular mortality, CHF and major CHD when compared with no TWA as well as minor TWA (Table 3).

Next, we sought to explore the differential effects of TWA based on anatomic localisation. At study enrolment, TWA were present in 1191 participants $(14.6 \%)$ in the anterior leads, 2150 participants $(26.3 \%)$ in the anterolateral leads and 607 participants $(7.4 \%)$ in the inferior leads (ESM Table 3 ). TWA in the anterolateral leads (I, aVL, V6) as well as inferior leads (II, III, aVF) were associated with higher risk of all-causemortality, cardiovascular mortality, $\mathrm{CHF}$ and major coronary artery heart disease, while TWA in the anterior leads (V2-V5) did not have statistically significant associations with cardiovascular events. None of the anatomic localisations had a significant impact on risk of stroke.

Then, we performed a secondary analysis to explore the possibility of a differential association of TWA with clinical cardiovascular events by the presence or absence of baseline CVD. The $p$ values for interaction did not meet criteria for statistical significance $(p>0.05)$, though the hazard associated with TWA was of numerically greater magnitude in those with prevalent CVD at baseline (ESM Table 4).

Finally, we found that TWA offered incremental value in discrimination of risk for major CHD when added to the UKPDS risk engine (Fig. 2). The area under the ROC curve was similar in those without CVD ( 0.6163 vs $0.6168, p$ value $0.96)$, but differed in participants with prevalent CVD $(0.5744$ vs $0.6030, p$ value 0.007$)$. This relationship was further clarified and the group without CVD had 649 individuals with events and 4729 without events (RI [reclassification index] [case] -0.41, RI [control] 0.55); NRI 0.14 (95\% CI 0.07, $0.22, p=0.0002$ ) (ESM Table 5). Those with previous CVD had 788 individuals with events and 1920 without events (RI [case] 0.03, RI [control] 0.22; NRI 0.24 (95\% CI 0.16, 0.32, $p<0.0001)$ (ESM Table 6).

\section{Discussion}

In this analysis of the ACCORD participants, we show that the risk of all-cause mortality, cardiovascular mortality, CHF and major CHD is increased in individuals with diabetes and TWA. Furthermore, we found that major TWA were associated with a higher risk than minor TWA.

In reviewing the body of literature, the presence of TWA is generally thought to convey a worse prognosis; however, many of these studies are limited to patients with previously known cardiac disease. In patients with a known left bundle branch block, the presence of TWA conferred a higher risk of developing heart failure within 30 days [10]. The prognostic role of TWA in ST elevation myocardial infarction patients 


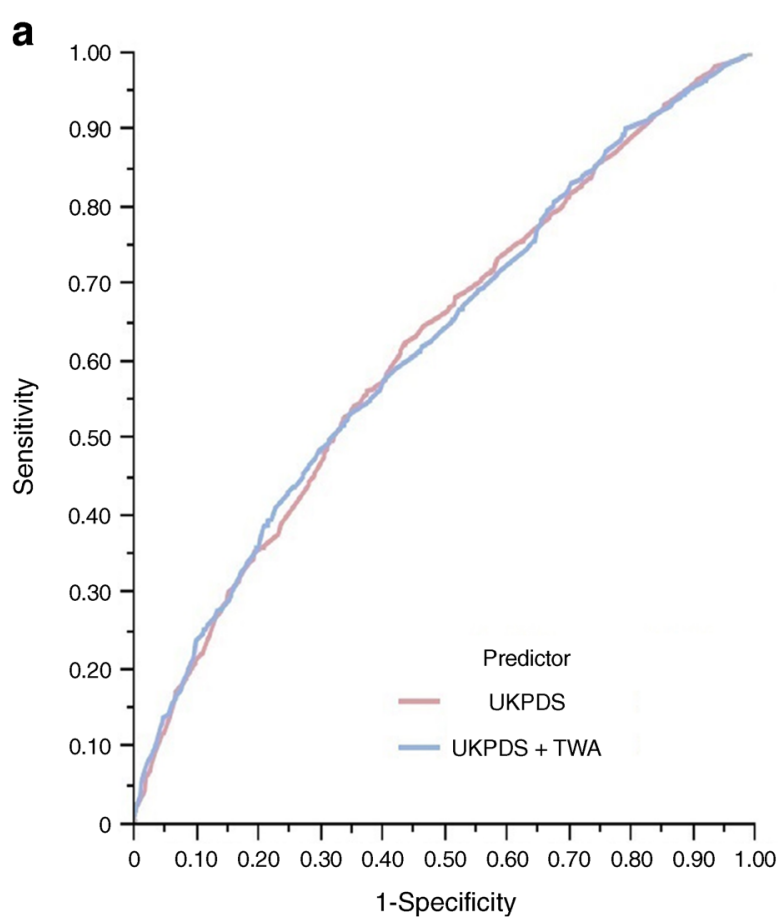

Fig. 2 ROC curve comparison of TWA in predicting CHD. (a) ROC curve of coronary artery disease prediction in patients without cardiovascular history. (b) ROC curve of coronary artery disease prediction in patients with cardiovascular history. In predicting CHD, area under ROC curves were similar for those without baseline CVD (AUC

has been evaluated as well. In a study investigating new myocardial infarctions, it was found that patients with both Q-wave and TWA had increased mortality at 30 days [11]. Those without Q-wave or TWA had decreased mortality and those that had either Q-wave or TWA were at intermediate risk of mortality. They also concluded that using TWA in ECG analysis for ST elevation myocardial infarction patients improves risk stratification [12]. This line of research further establishes the role that TWA may play in prognostication of long-term outcomes for patients.

There are a limited number of studies that have explored TWA in the general population without a history of CHD, and these studies are of smaller sample sizes. One such study $(n=$ 1814) included men who did not have known heart disease and were aged 42-60 and followed them for a mean of 21 years, finding that TWA was associated with increased CHD-related death as well as all-cause mortality [13]. However, other research found that TWA was thought to be associated with worse outcomes due to an increased association with ventricular arrythmia [14-17]. There have also been limited case reports describing TWA that developed in the setting of cardiogenic pulmonary oedema which resolved with the return to euvolaemia [18].

In our modelling of the TWA-outcomes relationship, our first model adjusted only for demographics (age, sex and race) and ACCORD treatment arm, while Model 2 added historical factors (prevalent CVD, smoking and years with diabetes),

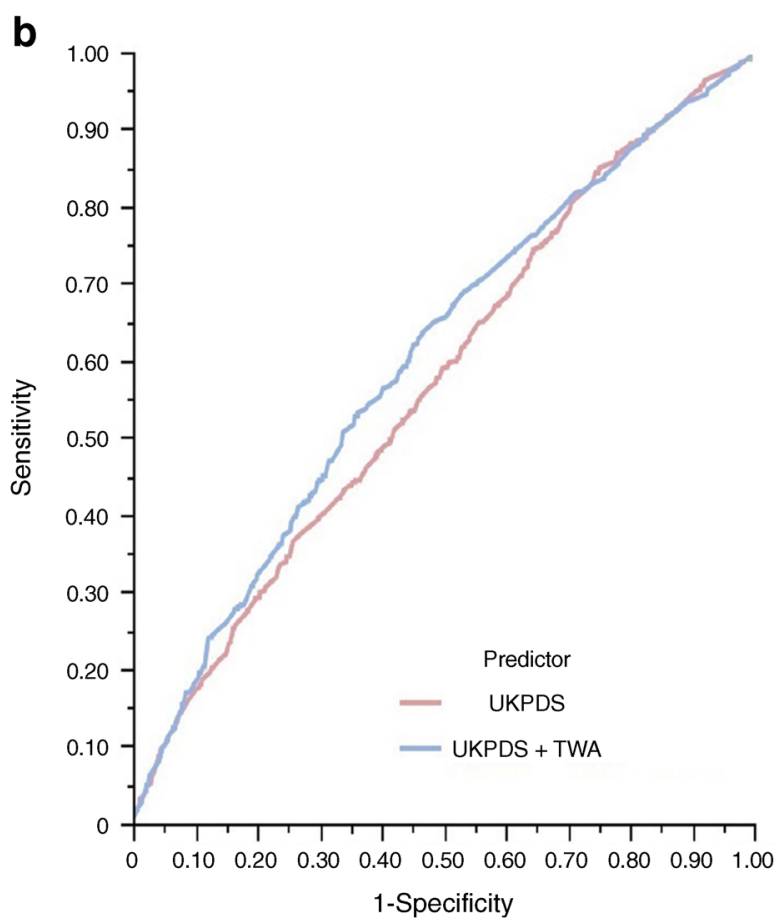

0.6163 vs 0.6168 , DeLong $p$ value for comparison of 0.96 ). In contrast, in those with prevalent CVD, TWA offered incremental value in the prediction of major CHD, with area under the ROC curves for the base model and the model with TWA included of 0.5744 and 0.6030 , respectively, with DeLong $p$ value for comparison of 0.0067

BMI and biochemical markers of disease, and Model 3 adjusted for ST segment abnormalities and major Q-wave. Model 1 likely provides the most generalisable data that might be useful in clinical practice, as it only adjusts for demographic variables that are known at the bedside. Model 2 controls for variables that can be ascertained from the medical record, demonstrating how TWA may have value if added to a risk prediction engine. Model 3 adjusts for other ECG abnormalities and is probably the model that is most specific in describing the risk associated with TWA that cannot be explained by any covariates, including concomitant ECG abnormalities.

In reviewing our analysis, we noted a dose-response type relationship between major and minor TWA. This result was consistent with our primary analysis, as the presence of TWA was associated with increased rates of mortality; it then stood to reason that major TWA would convey a significantly higher risk than minor TWA. This dose-response relationship is notable because the magnitude of TWA can then serve as a prognostic indicator of the degree of underlying cardiac pathology.

In general, when TWA are seen it is assumed to be caused by ischaemic events, either clinical or subclinical. It is then only reasonable that these TWA would be associated with increased mortality. However, this assumption is challenged by our data where anterior lead TWA were not associated with increased mortality. Given that this anterior distribution is supplied by the left anterior descending coronary artery, we 
had expected T-wave inversions in this distribution to be associated with significantly increased mortality. One possible explanation for our data is that TWA in anterolateral leads and inferior leads is representative of significant wall strain which in turn provides an increased risk of cardiovascular events. Another possible explanation for our result is that a normal, healthy vector of repolarisation is strongly positive inferiorly and laterally but is often mixed or transitioning in the anterior leads. Because these anterior forces are mixed, it would take lesser insults to change the repolarisation axis and result in TWA. Similarly we can conjecture that to overcome the significant positivity in inferior and lateral distributions would require a significant insult to these areas, such that participants with TWA in the anterolateral or inferior leads may have had a greater burden of ischaemic myocardium than those with TWA restricted to the anterior leads.

In regard to our secondary analysis, when we included an interaction term for TWA $\times$ CVD in our models, there was no statistically significant interaction for any outcomes, including all-cause mortality $(p=0.06)$, cardiovascular mortality $(p=$ $0.09)$, CHF $(p=0.81)$ or major CVD $(p=0.78)$. However, there was a global tendency for the presence of TWA to convey higher risk in those participants with prevalent CVD at baseline. While the interaction term did not meet criteria for statistical significance, we suspect that this relationship may be explained by the fact that TWA are a non-specific finding that may reflect underlying atherosclerotic CVD, or may represent a benign electroanatomic variant, ventricular hypertrophy or dispersion of repolarisation. In those with a history of CVD, it is more likely that the TWA are a 'true positive' and indicate underlying CVD.

The results of our final analysis are in agreement with this explanation, as TWA offered incremental value in discrimination of risk above and beyond that accounted for by the UKPDS risk engine. Furthermore, the results of the RI and NRI indicate that TWA may be useful in reclassifying risk in patients with and without prevalent CVD, although the magnitude of the NRI was greater in those with prevalent CVD. Similarly, we suspect that the fraction of participants with TWA that reflect true CVD is higher in the population with prevalent CVD. Therefore, it is not surprising that TWA offer more incremental value in discrimination of risk in participants with a history of CVD. It should be noted, however, that there are substantial differences between the study population of the UKPDS trial and the ACCORD trial, including an overall higher-risk population in the ACCORD trial, with a much longer history of years with diabetes. Thus, further studies assessing the incremental value of TWA should be conducted in patient populations with a more heterogeneous risk profile in the interest of maximising generalisability of the findings.

When reviewing the body of literature on TWA as predictors of adverse cardiovascular events, prior studies have utilised various definitions of TWA, including specific values of the MC that have varied between studies, Novacode, and manual measurements by investigators [19-22]. In addition, some analyses have lumped abnormalities of the ST segment together with TWA to create a construct of non-specific ST and T-wave abnormalities [23]. For our investigation, we wanted to focus on TWA while also allowing our research to be comparable to previous work. To accomplish this, we accounted for ST abnormalities in our multivariable model.

The principal strengths of our study are: (1) the large sample size; (2) uniform collection of data across multiple sites; (3) long follow-up through ACCORDION; and (4) unified ECG reviewing database. Limitations include the fact that there were significant differences in the baseline characteristics of those who had or did not have TWA. While we adjusted for clinically important covariates, the existence of residual confounding remains a possibility. There could also be a cohort effect whereby the prognostic impact of TWA among those with diabetes may be somewhat attenuated with the broadened therapeutic options used to treat diabetes in the 20 years since ACCORD began enrolment. Additionally, ACCORD inclusion criteria required participants to be at high cardiovascular risk, which limits the generalisability of our findings to individuals with diabetes and high cardiovascular risk, rather than all patients with diabetes.

Conclusions This study demonstrates that TWA are associated with an increased risk of subsequent clinical cardiovascular events and mortality in individuals with diabetes. This relationship was found to be the strongest in individuals with prior CHD but also exists in those without it.

Supplementary Information The online version contains peer-reviewed but unedited supplementary material available at https://doi.org/10.1007/ s00125-020-05337-8.

Acknowledgements This manuscript was prepared using research materials obtained from the NHLBI Biological Specimen and Data Repository Information Coordinating Center and does not necessarily reflect the opinions or views of the NHLBI.

Data availability The data that support the findings of this study are available from accordtrial.org but restrictions apply to the availability of these data, which were used under licence for the current study, and so are not publicly available. Requests for the data may be sent to the National Heart, Lung, and Blood Institute.

Funding This research received no specific grant from any funding agency in the public, commercial or not-for-profit sectors.

Authors' relationships and activities The authors declare that there are no relationships or activities that might bias, or be perceived to bias, their work.

Contribution statement MS and SM conceived and designed the analysis. MS performed the analysis. MS and SM interpreted the results. SM drafted the manuscript. ES, AB, PB, JY and MS revised the manuscript 
for critical intellectual content and assisted with interpretation of the results. All authors approved of the final manuscript for submission. MS is the guarantor of this work and, as such, had full access to all the data in the study and takes responsibility for the integrity of the data and the accuracy of the data analysis.

\section{References}

1. Aro A, Anttonen O, Tikkanen J et al (2012) Prevalence and prognostic significance of $\mathrm{T}$-wave inversions in right precordial leads of a 12-lead electrocardiogram in the middle-aged subjects. Circulation 125(21):2571-2577

2. Krittayaphong R, Muenkaew M, Chiewvit P, Ratanasit N, Kaolawanich Y, Phrommintikul A (2019) Electrocardiographic predictors of cardiovascular events in patients at high cardiovascular risk: a multicenter study. J Geriatr Cardiol 16(8):630-638. https://doi.org/10.11909/j.issn.1671-5411.2019.08.004

3. Jacob D, Main ML, Gupta S, Gosch K, McCoy M, Magalski A (2015) Prevalence and significance of isolated T wave inversion in 1755 consecutive American collegiate athletes. J Electrocardiol 48(3):407-414. https://doi.org/10.1016/j.jelectrocard.2015.03.005

4. Sheikh N, Papadakis M, Wilson M et al (2018) Diagnostic Yield of Genetic Testing in Young Athletes With T-Wave Inversion. Circulation. 138(12):1184-1194. https://doi.org/10.1161/ CIRCULATIONAHA.118.034208

5. Merlo M, Zaffalon D, Stolfo D et al (2019) ECG in dilated cardiomyopathy: specific findings and long-term prognostic significance. J Cardiovasc Med (Hagerstown) 20(7):450-458

6. McClean G, Riding NR, Pieles G et al (2019) Prevalence and significance of T-wave inversion in Arab and Black paediatric athletes: Should anterior T-wave inversion interpretation be governed by biological or chronological age? Eur J Prev Cardiol 26(6):641-652

7. Holkeri A, Eranti A, Haukilahti M et al (2019) Predicting sudden cardiac death in a general population using an electrocardiographic risk score. Heart

8. Stevens R, Kothari V, Adler A et al (2001) The UKPDS risk engine: a model for the risk of coronary heart disease in Type II diabetes (UKPDS 56). Clin Sci (Lond) 101(6):671-679. https://doi. org/10.1042/cs1010671

9. DeLong ER, DeLong DM, Clarke-Pearson DL (1988) Comparing the areas under two or more correlated receiver operating characteristic curves: a nonparametric approach. Biometrics. 44(3):837845. https://doi.org/10.2307/2531595

10. Jensen JR, Kragholm K, Bødker KW et al (2019) Association between $\mathrm{T}$-wave discordance and the development of heart failure in left bundle branch block patients: Results from the Copenhagen ECG study. J Electrocardiol 52:39-45. https://doi.org/10.1016/j. jelectrocard.2018.11.001

11. Koivula K, Nikus K, Viikilä J et al (2019) Comparison of the prognostic role of $\mathrm{Q}$ waves and inverted $\mathrm{T}$ waves in the presenting
ECG of STEMI patients. Ann Noninvasive Electrocardiol 24: e12585. https://doi.org/10.1111/anec.12585

12. Azab D, Zahran ME, Elmahmoudy A (2019) Initial $T$ wave morphology in the chest leads in patients presenting with anterior ST-segment elevation myocardial infarction and its correlation with spontaneous reperfusion of the left anterior descending coronary artery. Int J Cardiovasc Acad 5:52-57

13. Kurl S, Mäkikallio TH, Laukkanen JA (2015) T-wave inversion and mortality risk. Ann Med 47(1):69-73. https://doi.org/10.3109/ 07853890.2014 .985703

14. Yu Z, Chen Z, Wu Y (2018) Electrocardiographic parameters effectively predict ventricular tachycardia/fibrillation in acute phase and abnormal cardiac function in chronic phase of ST-segment elevation myocardial infarction. J Cardiovasc Electrophysiol 29(5):756766. https://doi.org/10.1111/jce. 13453

15. Tse G, Gong M, Wong WT et al (2017) The Tpeak - tend interval as an electrocardiographic risk marker of arrhythmic and mortality outcomes: A systematic review and meta-analysis. Heart Rhythm 14(8):1131-1137. https://doi.org/10.1016/j.hrthm.2017.05.031

16. Zagkli F, Georgakopoulou A, Chiladakis J (2019) QRS fragmentation and T-wave inversion as factors of vulnerability to recurrent ventricular tachycardia. Futur Cardiol 15(2):89-93. https://doi.org/ 10.2217/fca-2017-0104

17. Finocchiaro G, Papadakis M, Dhutia H et al (2019) Electrocardiographic differentiation between 'benign T-wave inversion' and arrhythmogenic right ventricular cardiomyopathy. Europace. 21(2):332-338. https://doi.org/10.1093/europace/ euy 179

18. Tampakis K, Makris N, Kontogiannis C et al (2018) Late T-wave inversion following resolution of non-ischemic acute pulmonary edema. Clin Case Rep 7(1):224-226

19. Bakhoya VN, Kurl S, Laukkanen JA (2014) T-wave inversion on electrocardiogram is related to the risk of acute coronary syndrome in the general population. Eur J Prev Cardiol 21(4):500-506. https://doi.org/10.1177/2047487312460022

20. Zhang Z, Prineas R, Soliman E, Baggett C, Heiss G (2012) Prognostic significance of serial Q/ST-T changes by the Minnesota Code and Novacode in the Atherosclerosis Risk in Communities (ARIC) Study. Eur J Prev Cardiol 19(6):1430-1436

21. Uberoi A, Sallam K, Perez M, Jain N, Ashley E, Froelicher V (2012) Prognostic implications of Q waves and T-wave inversion associated with early repolarization. Mayo Clin Proc 87(7):614 619. https://doi.org/10.1016/j.mayocp.2012.04.009

22. Cannon C, McCabe C, Stone P et al (1997) The electrocardiogram predicts one-year outcome of patients with unstable angina and non-Q wave myocardial infarction: results of the TIMI III Registry ECG Ancillary Study. J Am Coll Cardiol 30(1):133-140

23. Walsh J, Prineas R, Soliman E et al (2013) Association of isolated minor non-specific ST-segment and T-wave abnormalities with subclinical atherosclerosis in a middle-aged, biracial population: Coronary Artery Risk Development in Young Adults (CARDIA) study. Eur J Prev Cardiol 20(6):1035-1041. https://doi.org/10. $1177 / 2047487312460017$

Publisher's note Springer Nature remains neutral with regard to jurisdictional claims in published maps and institutional affiliations. 